INPLASY

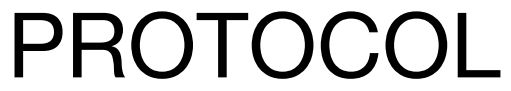

To cite: Zhao et al. Efficacy of Desferrioxamine Messylate in Intracalvarium Hematoma and Perihematomal Edema: A Systemic Review and MetaAnalysis. Inplasy protocol 2020100001. doi: 10.37766/inplasy2020.10.0001

Received: 30 September 2020

Published: 01 October 2020

Corresponding author: Mingfei Yang

iloveyoucmu@163.com

Author Affiliation:

Department of Neurosurgery, Qinghai Provincial People's Hospital, Xining, 810007, Qinghai, PR China

Support: Science of Qinghai.

Review Stage at time of this submission: Data analysis.

Conflicts of interest: N/A.

\section{Efficacy of Desferrioxamine Messylate in Intracalvarium Hematoma and Perihematomal Edema: A Systemic Review and Meta-Analysis}

Zhao, K1; Li, J2; Zhang, Q33 Du, CN4; Guo, Y5; Yang, MF6.

Review question / Objective: Intracalvarium hematoma and perihematomal edema(PHE) accompanying can both damage to the central nervous system in patients. Based on its pharmacological character of ferri ion chelating agent, numerous zooperies had proved that desferrioxamine messylate(DFO) would be used to treatment of intracerebral hemorrhage(ICH). We decided to perform a meta-analysis to find the evidence of application of DFO in clinical treatment. Condition being studied: Intracalvarium hematoma and perihematomal edema (PHE).

Information sources: Three open electronic database of PubMed, Embase and Cochrane.

INPLASY registration number: This protocol was registered with the International Platform of Registered Systematic Review and Meta-Analysis Protocols (INPLASY) on 01 October 2020 and was last updated on 01 October 2020 (registration number INPLASY2020100001).

\section{INTRODUCTION}

Review question / Objective: Intracalvarium hematoma and perihematomal edema(PHE) accompanying can both damage to the central nervous system in patients. Based on its pharmacological character of ferri ion chelating agent, numerous zooperies had proved that desferrioxamine
messylate(DFO) would be used to treatment of intracerebral hemorrhage(ICH). We decided to perform a meta-analysis to find the evidence of application of DFO in clinical treatment.

Rationale: Studies were identified after literature search from PubMed, Embase and Cochrane up to June 2, 2020, which 
included the data extraction of difference of National Institute of Health stroke scale(NIHSS) scores, hematoma volume(HV) and relative PHE(RPHE) defined that PHE volume was divided by HV or the total volume of hematoma and PHE. The standard mean difference(SMD) and 95 percent confidence interals $(95 \% \mathrm{Cl})$ were calculated by random effects model. Isquare(12) statistic was used to test the heterogeneity.

Condition being studied: Intracalvarium hematoma and perihematomal edema (PHE).

\section{METHODS}

Search strategy: (("Deferoxamine"[Mesh]) OR / ( ( ( / ( / ( ( / ( ( (Desferal[Title) Abstract]) OR("Methanesulfonate, Defe $r o x$ a m i n e " [ T t le / Abstract])) OR ("Deferoxamine Methanesulfonate "[ Title / Abstract])) OR (" Mesy late, Deferoxamine"[Title/Abstract])) OR("Deferoxamine Mesylate"[Title/ A b s t ract ])) OR ("M e silate, Defe $r o x a m$ i ne "[ T i t le / Abstract])) OR ("Deferoxamine Mesilate"[Title/Abstract]))OR ("Mesylate, Desferrioxamine B "[Title/ Abstract]))OR("Desferrioxamine $B$

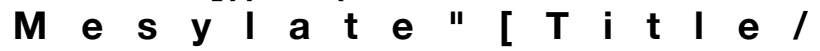
Abstract]))OR("Desferroxamine"[Title/ Abstract]))OR("Deferrioxamine B"[Title/ Abstract])) OR(Deferoximine [Title/ Abstract]))OR("Desferrioxamine B" [Title/ Abstract]))OR("Deferoxamine B"[Title/ Abstract]))OR (Desferioximine[Title/ Abstract]))OR(Desferrioxamine[Title/ Abstract ]) ) ) AND ( "Cerebra I Hemorrhage"[Mesh])OR(()(()(()(()("Hemorrh age *, Cerebral Brain "[ Title / Abstract]) OR ("Cerebral Brain Hemorrhage*"[Title/Abstract]))OR("Brain Hemorrhage*, Cerebral "[Title / Abstract])) OR (" Hemorrhage *, Cerebral" [Title/Abstract]))OR("Cerebral H e m or $r h$ a g e $s$ "[ T i t l e / Abstract])) OR ("ICHs "[ Title / Abstract])) OR ("Hemorrhage*, Intracerebral "[Title/Abstract])) OR("Hemorrhage, Intracerebral"[Title/
A b s tract ]) ) OR ( "I C H "[ Tit I e / Abstract]))OR("Parenchymal Hemorrhages, Cerebral"[Title/Abstract]))OR("Parenchymal Hemorrhage, Cerebral"[Title/Abstract])) OR ( "Hemorrhage *, Cerebral Parenchymal"[Title/Abstract]))OR("Cerebral Parenchymal Hemorrhage *[Title/ Abstract ])) OR ( "He morrhage *, Cerebrum"[Title/Abstract]))OR("Cerebrum Hemorrhage*"[Title/Abstract]))).

Participant or population: People participated in experiment in all of studies, age range were 39 to 81 with male majority, were 239 patients with the etiology of craniocerebral trauma(47) and hemorrhagic stroke(192).

Intervention: Desferrioxamine messylate used in patients.

Comparator: Non-desferrioxamine messylate used in patients.

Study designs to be included: Random control trials.

Eligibility criteria: (1)Some features of articles were not restricted absolutely: language of articles, regions and etiologies of intracalvarium hematoma; (2)Date of publication was up to June 2, 2020; (3)Types of studies were not restricted except that the objects of studies were belong to animals; (4)Several types of articles were deleted directly: reviews, meta-analysis, letters, comments and protocols; (5)They were sensible to be omitted that articles with titles or abstracts which were not in accord with the title of our meta-analysis and publications with duplication.

Information sources: Three open electronic database of PubMed, Embase and Cochrane.

Main outcome(s): Difference of National Institute of Health stroke scale(NIHSS) scores, hematoma volume(HV) and relative PHE(RPHE) defined that PHE volume was divided by $\mathrm{HV}$ or the total volume of hematoma and PHE. 


\section{Additional outcome(s): N/A.}

Data management: Mean and standard deviation(SD) were used to perform statistical description of continuous variables which obeyed normal distribution. Statistical difference of data before remedy between intervention and control groups would be tested by the module of one way Analysis of Variance(ANOVA) in a statistical software named SigmaStat version 4.0. If there was no statistical difference, data after remedy could be directly used to meta-analysis. Or manual calculation would be implemented to the differences of data before and after treatment.

Quality assessment / Risk of bias analysis: The quality assessment of literature included was performed via the Cochrane Collaboration's Tool of Assessing Risk of Bias by the software Review Manager 5.3 after data extraction.

Strategy of data synthesis: Meta-analysis was completed through corresponding modules in Software for Statistics and Data Science (version 15.1; College Station, Texas 77845 USA). The standard mean difference(SMD) and 95 percent confidence intervals $(95 \% \mathrm{Cl})$ were calculated by random effects model. I-square(I2) statistic was used to test the heterogeneity. All $p$ values were two-side with a significant level at 0.05 .

\section{Subgroup analysis: N/A.}

Sensibility analysis: Sensitivity analysis, each study was omitted one by one and retrieved the pooled HR of the rest studies or the random effects model was switched to fixed effects model, was the way to checkout the stability of overall results. Funnel plot asymmetry and Egger's regression were used to detect publication bias. Two methods were used to attenuate heterogeneity and enhance sensitivity: deletion of studies with publication bias and analysis of different 12 of overall SMD of the rest studies that everyone in quondam studies was omitted one by one. All $p$ values were two-side with a significant level at $\mathbf{0 . 0 5}$.
Language: No.

Country(ies) involved: USA, PR China.

Other relevant information: N/A.

Keywords: Perihematomal Edema, Intracalvarium Hematoma, Desferrioxamine Messylate, Meta-analysis, Treatment.

Dissemination plans: Published on Scientific Reports.

Contributions of each author: Yang, M.F. and Zhao, K. conceived the study. Zhao, K. and Guo, Y. selected reports and extracted the data. Du, C.N., and $\mathrm{Li}, \mathrm{J}$. analysed and interpreted the data. Zhao, K. and Zhang, $Q$. wrote the first draft of the manuscript.

Author 1 - Kai Zhao.

Author 2 - Jing Li.

Author 3 - Qiang Zhang.

Author 4 - Chaonan Du.

Author 5 - Yu Guo.

Author 6 - Mingfei Yang. 